\begin{tabular}{|c|c|c|}
\hline \multirow{2}{*}{ IDUNAS } & NATURAL \& APPLIED SCIENCES & 2021 \\
& Jol. 4 \\
No. 1 \\
$(30-37)$
\end{tabular}

\title{
An Image Segmentation Method for Wound Healing Assay Images
}

\author{
Research Article

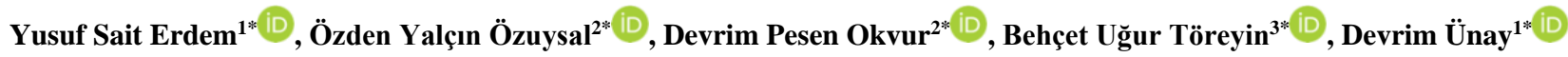 \\ ${ }^{l}$ Department of Electrical and Electronics Engineering, İzmir Demokrasi University, Karabağlar, İzmir, Turkey. \\ ${ }^{2}$ Department of Molecular Biology and Genetics, İzmir Institute of Technology, Urla, İzmir, Turkey. \\ ${ }^{3}$ Istanbul Technical University Informatics Institute, İTÜ Ayazağa Kampüsü, Sarıyer, İstanbul, Turkey. \\ Author E-mails: \\ yusufsaiterdem@gmail.com \\ ozdenyalcin@iyte.edu.tr \\ devrimpesen@iyte.edu.tr \\ toreyin@itu.edu.tr \\ devrim.unay@uzem.idu.edu.tr \\ *Correspondance to: Yusuf Sait Erdem, Department of Electrical and Electronics Engineering, İzmir Demokrasi University, \\ Karabağlar, İzmir, Turkey. \\ DOI: 10.38061/idunas.853356
}

Received: 04.01.2021; Accepted: 05.04.2021

\section{Abstract}

Wound healing assays are important for molecular biologists to understand the mechanisms of cell migration. For the analysis of wound healing assays, accurate segmentation of the wound front is a necessity. Manual annotation of the wound front is inconvenient since it is time-consuming and annotatordependent. Thus automated, fast, and robust solutions are required. There are several image processing techniques proposed to fulfill this need. However, requirement for specification of optimal parameters, the need for human intervention, and the lack of high accuracy emerge as the downfalls for most of them. In this study we have proposed a novel method to overcome these difficulties.

Keywords: Image segmentation, quantification, wound healing assay, collective cell migration, phasecontrast microscopy.

\section{INTRODUCTION}

Wound healing assay in vitro became a widespread technique to analyze many complicated biological processes such as immune system activation, spread of tumor, and metastasis as well as embodiment of tissues. Advances in the automation of microscopic imaging has boosted extraction of more data from wound healing assays [4, 6-7]. Segmentation of such excessive amount of data by hand is tedious, time consuming, and subjective. Therefore, many automated methods for the segmentation of wound healing assay images are proposed in the literature. However, the need for more accurate, robust, and practical methods still resides. 
There are several widely used tools available for the segmentation of wound healing assays such as MRI Wound Healing Tool macro developed for ImageJ [10] and Cell 1 Image Velocimetry (CIV) [9]. In the MRI Wound Healing Tool plugin, users should tune the parameters such as the preferred method, radius of structuring element used in morphological opening, and minimum wound size. In the segmentation method of CIV, parameters such as scaling factor, radius of local contrast normalization, thresholds for local and global filtering, and kernel size should be set by the user to obtain an adequate segmentation result.

Deep learning methods are also utilized for wound healing assay image segmentation [11]. While these methods may maintain high accuracy, they need a training process with a decent amount of annotated data.

Matsubayashi et al. introduced 'White wave' tool which suggests a novel measure to take account instead of direct frontier distances for wound closing analysis [1]. The tool first takes difference image of consecutive frames of wound closing footage. The difference image is computed by taking absolute value of difference of each corresponding pixel. Then calculates a histogram of resulting difference image's vertical average intensities corresponding to $\mathrm{x}$-axis. ' $\mathrm{x}$ ' coordinates of two peaked average intensity values of histogram are taken as positions of wound edges assuming that the wound closing image has two vertical sides of wounds. Matsubayashi et al. proposes that average value is more robust and contains more information than direct frontier distances. That average value represents the $\mathrm{x}$ location of wound edge, shows movement of wound sides even after complete closure of the wound which is claimed that is more consistent to the domain knowledge [1]. Even though this method unveils movement information, it does not produce data to calculate an actual wound closure speed metric nor a wound area metric.

The TScratch wound analysis tool published by Gebäck - Tobias et al. uses discrete curvelet transformation together with morphological operations to segment wound surfaces [2]. Curvelet transformation is used for detection of edges and elimination of noises in a wound closing assay image. At first, curvelet magnitude image is generated by using 2 scale levels where wound surface edges are visible. Then morphological opening is applied. Two peak areas on curvelet histogram are taken as true edges and the rest is assumed as noise edges. Small areas are considered as noise and removed on curvelet transformed image after a morphological opening operation. Finally, an erosion operation is applied to resulting image [2]. TScratch method finds scale-level parameter automatically but that critical parameter is needed to be empirically adjusted by user on same cases for good segmentation result.

Suarez-Arnedo et al. developed a wound healing assay analysis tool by using contrast enhancement and variance filtering [3]. The tool takes radius of variance filter, binarization intensity threshold and saturation percentage as user inputs for each frame in video input. The tool first enhances contrast using saturation percentage parameter than applies variance filter. Thresholds image for binarization. Then applies erosion to remove small, independent particles and hole filling operation. Resulting, largest component is taken as the empty wound area. Width deviation of the wound is calculated by taking horizontal distances across the detected wound edges. The tool also calculates the wound area in pixels as well as the empty wound area to cell edge area ratio [3].

A high throughput wound healing assay method for quantitative output is suggested by Zordan et al. where an instrument for reproducible wounding and entropy filtering for segmentation is used. The bright field image collected from the assay is cropped using a user defined input to omit some unnecessary regions. An 11x11 pixel sized entropy filter is applied and thresholded with an input value of between 0.5 and 0.85 , generally. 2 The resulting binary image is inverted, and a morphological opening operation is applied to remove small wound regions. Dilation is applied after for smooth surfaces. To generate a connected wound area, a morphological closing applied with a 5x5 pixel sized oval kernel and a morphological fill is applied. 
Detected cell regions those are touching to single border of the image are discarded. Area of the empty wound region on entropy filtered image is used to calculate wound closing percentage during the assay [4].

Topman et al. calculated wound area by segmenting wound assay images using two separate standard deviation windows [5]. One of these windows has "big" size for detecting large shapes and the other one has small size for keeping small details on the edges of cell regions. After standard deviation is applied with "big" windows, a peak point in histogram of intensities is detected. Half of this intensity value is used as threshold value for the resulting image. After obtaining the binary image, dilation is applied by a rectangular kernel whose size is half of the big window size. Same procedure is applied with the "small" windows size except dilation and the resulting images are combined. Morphological closing and opening operations applied to combined image using kernel with size of the small window. Since the sizes of small and big windows are determined as constant values in real metrics, real scale of the assay images should be known in this method. Garcia et al. introduced a similar method, but they have used a single window for standard deviation and morphological filling operation additionally [8].

These methods work successfully under certain conditions but, they depend on many critical parameters such as kernel size [3, 5, 8], saturation rate [3-4], and scale-level [2]. To achieve high and consistent accuracy on various conditions, they require human intervention for the determination of optimal parameter set, which makes them difficult to use. Our method, on the other hand, depends on a single parameter and thus emerges as a more robust solution. Even if this single parameter of ours needs to be reset to obtain premium accuracy on a limited number of cases, it can be approximately determined by analyzing the width of cell pixels present in the assay.

\section{METHODOLOGY}

\section{Experimentation and Data}

The experiments and the image acquisition of the in-vitro wound healing assays employed in this study are realized at the Molecular Biology and Genetics Department of İzmir Institute of Technology Non-invasive breast cancer cell line MCF7 cells in epithelial morphology are planted in 6-well petri dishes and allowed to attach for 24 hours. The wound was artificially created prior to imaging by scraping the surface in a line with the tip of a yellow pipette. For the imaging petri dishes are placed in the incubation room of the Leica SP8 microscopy system providing constant temperature of $37^{\circ} \mathrm{C}$ and $5 \% \mathrm{CO} 2$. Phasecontrast image acquisition is performed using a Leica SP8 microscopy system with 10x objective at every 60 minutes throughout a 48-hour duration. The images acquired have 1920x1440 pixels resolution.

\section{Manual Segmentation}

Wound healing assay data is annotated manually for the evaluation of the methods. Contour of each empty wound region is annotated as polygons using the online image annotation tool Supervisely [12]. Annotations are realized by a single annotator under 3 the supervision of molecular biologists. For this study, four wound healing assay videos are annotated, corresponding to a total of 65 frames in 1920x 1440 pixel resolution. Number of annotated frames per assay varies depending on the wound closure speed.

\section{Automated Segmentation of Wound Healing Assays}

Our method at first takes spatial derivative of the 2D input image. Then it applies smoothing by convolving the difference image with Gaussian kernel and binarizes the result by nonzero pixels. Width of the Gaussian kernel is a constant parameter which is loosely equal to the width of the smallest cell in input 
images. For our experiments, 25 pixels is taken as smallest cell width in pixels. Small blobs are removed after dilating the resulting image, and erosion is applied on the outcome. Isolated cell areas touching a single border of the image are eliminated, as in Zordan et al.'s method [4], since they do not belong to any side of the wound. The biggest detected empty area assumed to be the open wound area. Following the segmentation of the first frame, consecutive frames are analyzed in a similar manner except, not only the biggest detected empty area is returned but other empty regions are taken in account too. Remaining empty regions are sorted according to their areas. To determine these regions as empty region, shrinkage rate of the biggest region is calculated by dividing current biggest empty area with the previous one. This shrinkage rate is multiplied by current biggest empty area. Regions having larger size than the calculated area are assigned as the wound region if the total area is less than the one before as shown in Figure 1.

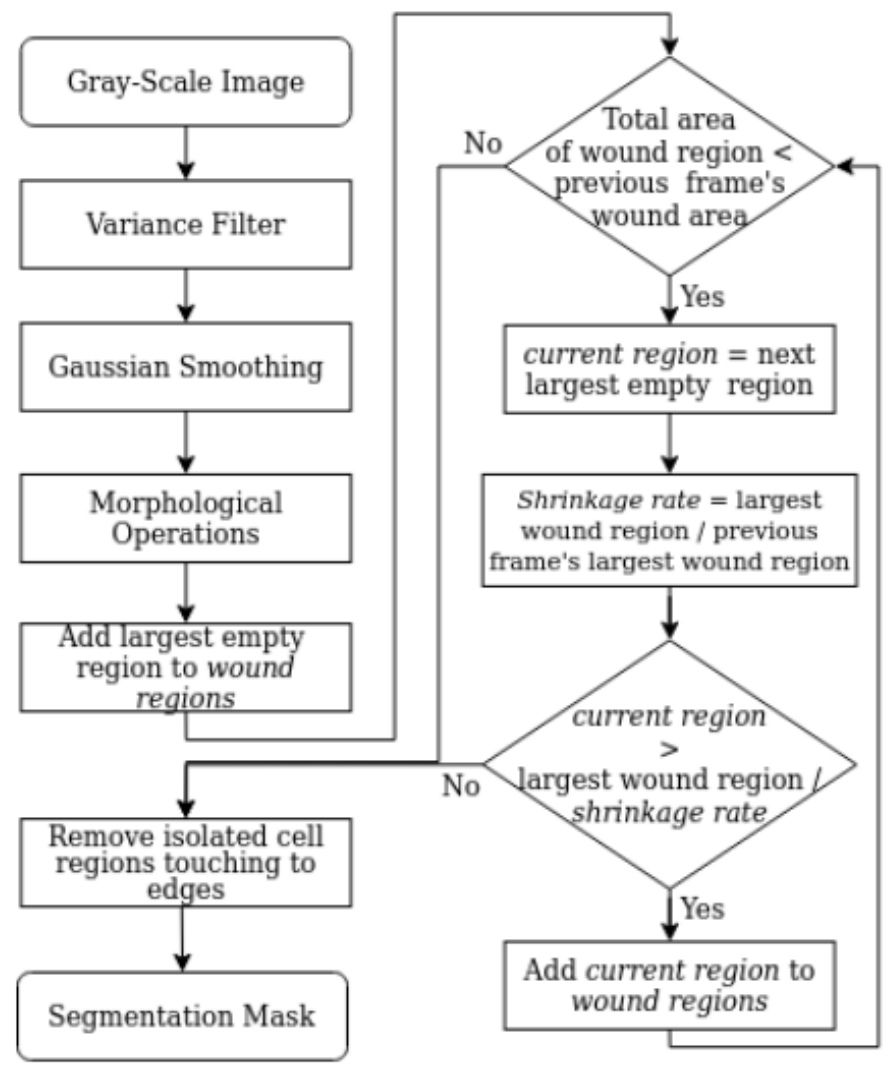

Figure 1. Flowchart of our image segmentation method for wound healing assay images.

Once the wound image is segmented, the two opposite borders (wound fronts) of the wound opening are extracted. For that purpose, a bounding box is fitted to the segmented wound opening and split into two vertical halves using a midline extraction approach as shown in Figure 2.
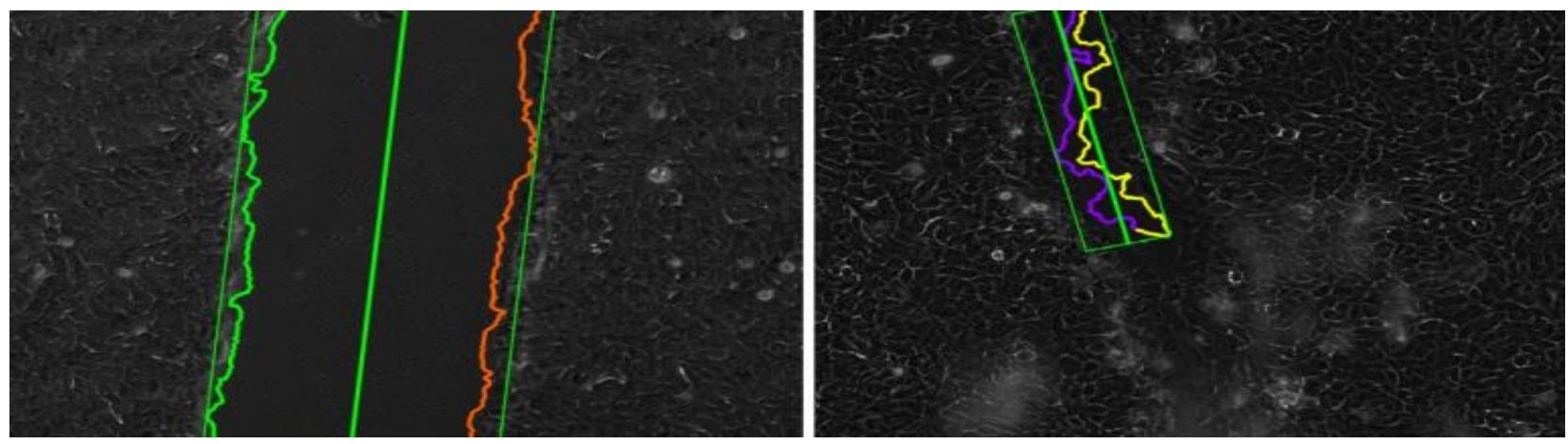

Figure 2. Identification of the wound fronts or borders. 


\section{Metrics Used for Quantification}

For the evaluation of the segmentation accuracy obtained by the methods experimented, Dice coefficient score is employed as:

DiceScore $\left(R_{g}, R_{p}\right)=\frac{2 *\left|R_{g} \cap R_{p}\right|}{\left|R_{g}\right|+\left|R_{p}\right|}$

where $\mathrm{Rg}$ and $\mathrm{Rp}$ correspond to the ground truth and predicted regions, respectively. To quantify wound closure from the assay data, the Mean Surface Distance is calculated on the detected wound surfaces at each frame as well as the rate of the area occupied by the open wound region. Mean Surface Distance measures the distance between two surfaces by averaging the minimum distances across surfaces based on the following formula:

$\operatorname{MSD}(S 1, S 2)=\frac{1}{N_{S 1}+N_{S 2}}\left(\sum_{i=1}^{N_{S 1}}\left|d_{i}^{S 1 \rightarrow S 2}\right|+\sum_{i=1}^{N_{S 2}}\left|d_{i}^{S 2 \rightarrow S 1}\right|\right)$

where S1 and S2 are the frontiers of wound regions, NS1 and NS2 are the point counts of the surfaces, and $\mathrm{d}$ is the 2D Euclidean distances.

\section{EXPERIMENTAL RESULTS AND DISCUSSION}

We have compared the performance and robustness of our method with other automated solutions present in the literature using four annotated wound healing assay data. Figure 3 demonstrates exemplary frames from one of the assays together with the corresponding ground truths and the related Dice scores of the methods per frame. As observed in the graphical result (Figure 3, top), all methods show high accuracy in the beginning of the experiment (i.e. at the initial frames) while as the wound healing occurs (i.e. towards later frames) accuracies of the methods decline. We see that TScratch is the worst performer overall, whereas our method achieves similar performance overall with PyScratch. Figure 4 graphically presents the average Dice scores of each method, with the corresponding standard deviations, over all four annotated essays. As seen, our method outperforms its rivals in average Dice score. We observe that all methods depict large standard deviations, which can be attributed to the performance variations due to the increased difficulty in wound segmentation as the healing progresses. Only Topman et al.'s method has a slightly lower standard deviation than our's, but that method has the lowest average dice score. 

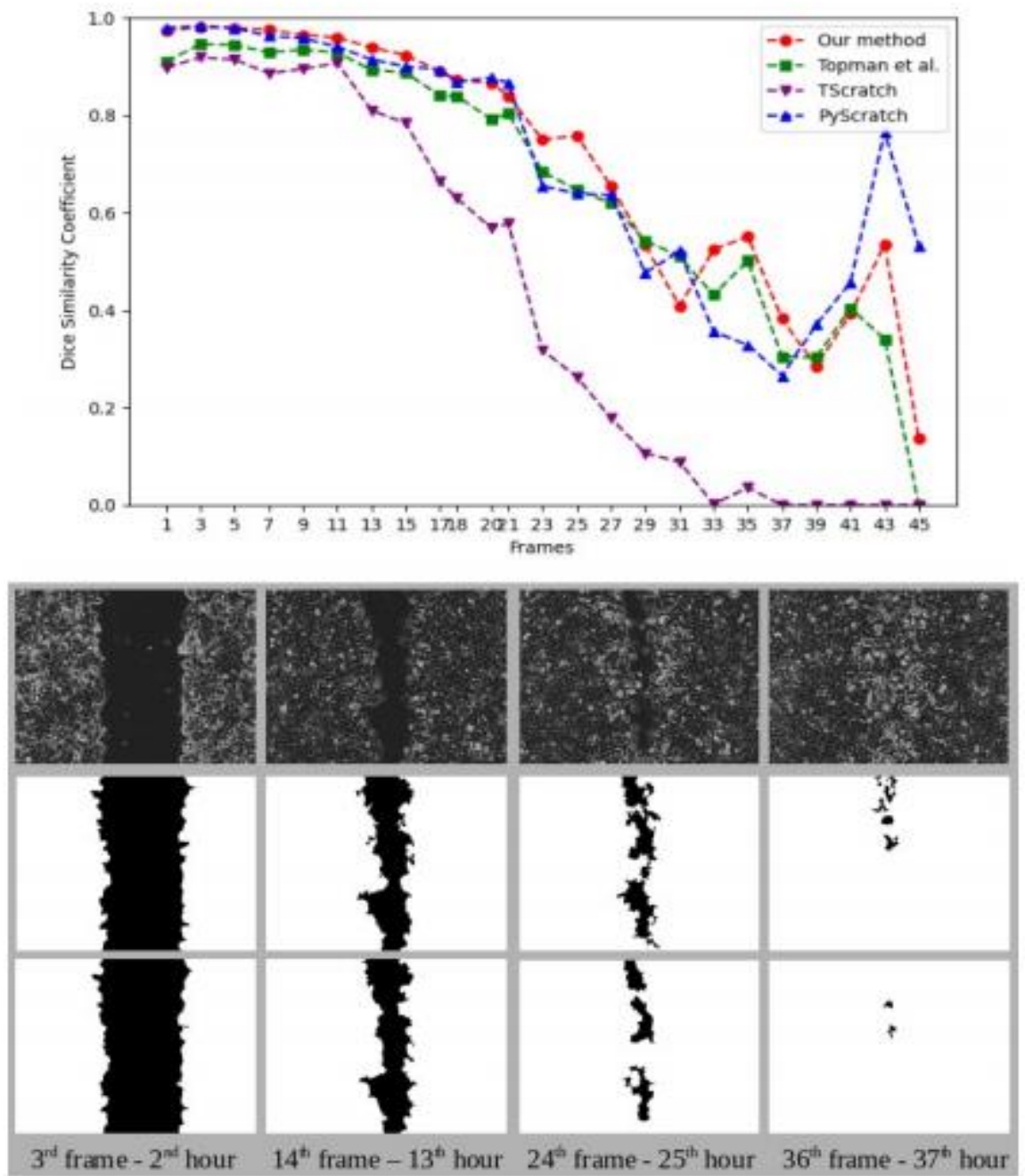

Figure 3. Top: Performance comparison of the methods based on their Dice similarity coefficients per frame. Second row: Exemplary frames from a wound healing assay data. Third row: Corresponding ground truth segmentations. Fourth row: Corresponding segmentation results of our proposed method.

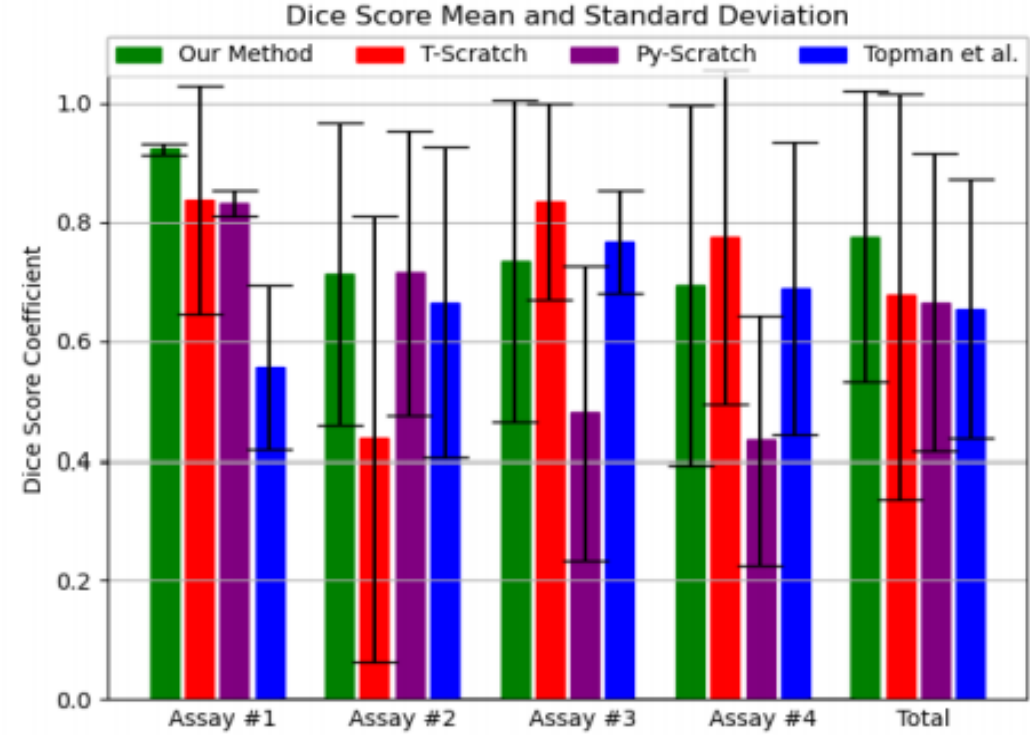

Figure 4. Performance comparison of the methods based on their average Dice similarity coefficients and standard deviations per assay as well as overall (i.e. Total). 
The wound surface distance together with the area of the open wound region is computed and displayed for each frame to allow for better representation of the wound healing. Figure 5 shows a visual example of the distances computed from the opposite wound fronts (left) and the temporal change in the mean surface distance (right). We see 6 gradual decline in the mean surface distances of the wound opening, which can be attributed to wound repair.
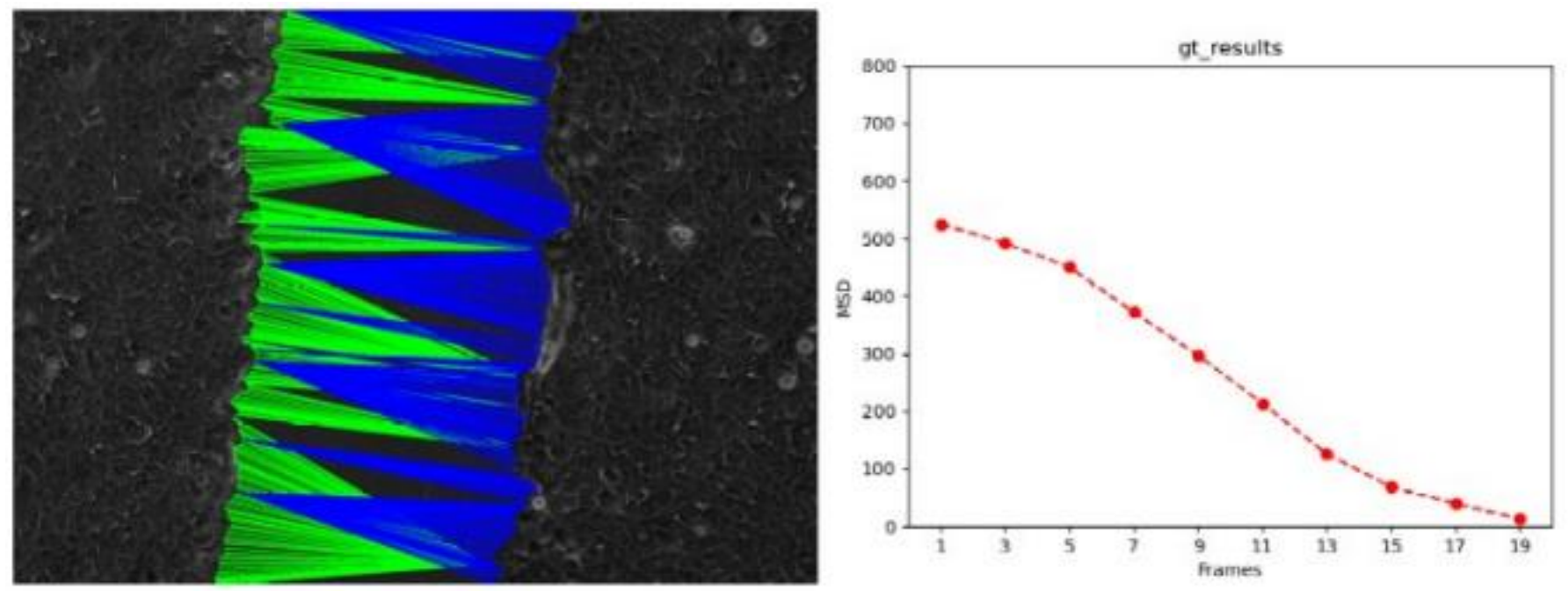

Figure 5. Left: Visualization of closest surface distances. Right: Mean surface distance graph of a wound healing assay.

\section{CONCLUSION}

Wound healing assays are frequently employed by molecular biologists to study collective cell migration and cell-cell interaction. Routinely, these assays are continuously monitored by optical microscopy and image series are generated for their further analyses. Segmentation of the wound area from these image series is a critical step for the analysis and evaluation of the assay results. As manual segmentation is tedious, time consuming and subjective, automated solutions are needed. For this purpose, in this study we prosposed an automated, robust, and accurate solution for segmenting wound healing assay data. Comparison with the state-of-the-art methods, as quantified on several expert-annotated real wound healing assay data using Dice overlap and surface distance metrics, showed that our proposed solution achieves higher and consistent accuracies, and thus emerges as a suitable alternative for the quantitative analysis of in-vitro wound healing assays.

\section{ACKNOWLEDGMENTS}

This work is supported by the Scientific and Technological Research Council of Turkey (Tübitak) under grant no 119E578.

\section{REFERENCES}

1. Matsubayashi, Y., William R., Paul M. (2011)., White wave analysis of epithelial scratch wound healing reveals how cells mobilise back from the leading edge in a myosin-II-dependent fashion., Journal of cell science 124.7: 1017-1021.
2. Gebäck, T. (2009). et al., TScratch: a novel and simple software tool for automated analysis of monolayer wound healing assays: Short Technical Reports., Biotechniques 46.4: 265-274. 
3. Suarez-Arnedo, A. (2020). et al., An image J plugin for the high throughput image analysis of in vitro scratch wound healing assays, bioRxiv.

4. Zordan, Michael D. (2011). et al., A high throughput, interactive imaging, bright-field wound healing assay., Cytometry Part A 79.3: 227-232.

5. Topman, G., Orna S., and Amit G. (2012). A standardized objective method for continuously measuring the kinematics of cultures covering a mechanically damaged site., Medical engineering \& physics 34.2 : 225-232.

6. Grada, A. (2017( et al., Research techniques made simple: analysis of collective cell migration using the wound healing assay., Journal of Investigative Dermatology 137.2: e11-e16.

7. Huang, K., and Robert F. M. (2004). From quantitative microscopy to automated image understanding., Journal of biomedical optics 9.5: 893-913.

8. Garcia, F., Vladimir Fernanda G., and B. de Jesus M. (2020). PyScratch: an ease of use tool for analysis of Scratch assays., Computer Methods and Programs in Biomedicine: 105476.

9. Milde, F. (2012). et al., Cell Image Velocimetry (CIV): boosting the automated quantification of cell migration in wound healing assays., Integrative Biology 4.11: 1437-1447.

10. Wound healing image segmentation tool: http://dev.mri.cnrs.fr/projects/imagejmacros/wiki/ Wound_Healing_Tool

11. Mayal1, B. (2020). et al., Automated Analysis of Wound Healing Microscopy Image Series-A Preliminary Study., 2020 Medical Technologies Congress (TIPTEKNO). IEEE.

12. Online image annotation tool: https://supervise.ly 